Article

\title{
Energy Signal-Aided Secure Beamforming and Self-Energy Recycling in Full-Duplex Wireless-Powered Relay Networks
}

\author{
Lizhen Ou ${ }^{1,+}$, Peipei Chen ${ }^{1, *,+}$, Xueshan Luo ${ }^{1}$, Shuping Dang ${ }^{2}$ and Yuchen Sun ${ }^{1}$ \\ 1 Science and Technology on Information Systems Engineering Laboratory, National University of Defense \\ Technology, Changsha 410073, China; oulizhen@nudt.edu.cn (L.O.); xsluo@nudt.edu.cn (X.L.); \\ sunyuchen18@nudt.edu.cn (Y.S.) \\ 2 Department of Electrical and Electronic Engineering, University of Bristol, Bristol BS8 1UB, UK; \\ shuping.dang@bristol.ac.uk \\ * Correspondence: chenpeipei20@nudt.edu.cn \\ + These authors contributed equally to this work.
}

Citation: Ou, L.; Chen, P.; Luo, X.; Dang, S.; Sun, Y. Energy Signal-Aided Secure Beamforming and Self-Energy Recycling in Full-Duplex Wireless-Powered Relay Networks. Energies 2021, 14, 6497. https:// doi.org/10.3390/en14206497

Academic Editor: Sangheon Pack

Received: 21 August 2021

Accepted: 3 October 2021

Published: 11 October 2021

Publisher's Note: MDPI stays neutral with regard to jurisdictional claims in published maps and institutional affiliations.

Copyright: (c) 2021 by the authors. Licensee MDPI, Basel, Switzerland. This article is an open access article distributed under the terms and conditions of the Creative Commons Attribution (CC BY) license (https:// creativecommons.org/licenses/by/ $4.0 /)$.

\begin{abstract}
In this paper, the object of study is secure transmission and green energy transfer in fullduplex (FD) wireless-powered relay (WPR) secure systems, where an FD relay collects the power from radio-frequency signs and transmits the information in the face of multiple eavesdroppers. In order to improve the efficiency and safety of the contemporaneous wireless energy and information delivery, we propose a joint energy-signal- (ES-) aided secure beamforming and time-switch scheme under the self-power circulation protocol at the relay. The question formulated in this paper is to maximize the confidentiality rate according to energy restrictions at both the relay and energy receiver. As the question is non-salient and hard to resolve directly, we transform it into two sub-problems. For the first sub-problem, a two-level optimization technique is suggested to separately gain the optimal beamforming as well as the ES covariance. The extrinsic rank is a single-variable majorization question, which can be solved by single-dimensional (1D) examination. We attain an optimal solution to the inner level by a semi-definite relaxation (SDR) technique. For the second sub-problem, we again use 1D search to solve this problem. Moreover, we prove that SDR always exists as a level-1 optimal resolution. Mathematical outcomes show that the suggested plan can achieve a considerable gain of confidentiality rate by comparison with other benchmark plans.
\end{abstract}

Keywords: physical layer security; power collecting; contemporaneous wireless information and energy transfer

\section{Introduction}

Collecting power from RF signs is regarded as a promising approach to extend the longevity of power-forced wireless systems, especially concerning the high-power depletion of 5G wireless networks [1].

Meanwhile, contemporary wireless information and power transfer (SWIPT) can be realized by RF signals. The main purpose of SWIPT is to extend the longevity of wireless-powered systems and has drawn much attention after operating in various wireless communication fields, such as multiple-input single-output (MISO) [2,3], multipleinput-multiple-output (MIMO) [4], one-way amplify-and-forward (AF) relay systems [5,6], and two-way AF relay systems $[7,8]$.

However, FD relaying produces loopback-interference (LI) limits that could degrade secrecy performance. Although many self-interference elimination techniques [9,10] have been proposed to eliminate self-interference, the implementation of these techniques is at the expense of expensive hardware and additional energy consumption. An effective relay-based self-energy recycling scheme [11] has been put forward to cope with the harmful effects of LI [12]. Regarding relaying systems, the joint secure beamforming scheme $[13,14]$ and antenna distribution scheme [15] are applied to self-energy recycling 
(S-ER). In addition, Reference [16] expands on [12,15], applying the S-ER strategy to the FD source for self-energy recycling and confidential information transmission, and advancing the optimal beamforming scheme. Moreover, in [17], a fresh two-stage protocol was suggested in which the relay works in an FD pattern for utilizing self-energy.

However, the secure transmission approaches have not taken into account FD wirelesspowered relay (FD-WPR) systems. In addition, the main challenge of FD-WPR secure systems is that a relay can harvest energy when transmitting signals, but this parallelism could lead to increasing LI and information leakage. Unfortunately, these issues have not been well addressed in the existing literature to the best of our knowledge.

Motivated by these open issues, we investigated secure transmission and renewable energy harvesting at mobile receivers in FD-WPR secure systems. To avoid the influence of LI and harvest energy overheard loopback interference arising from the FD relay, we designed a two-phase self-energy recycling protocol. The relay emits an energy signal (ES) to prevent information from being stolen by eavesdropping nodes [5]. The ES is a pre-designed pseudo-random signal that is conducive to secrecy information transmission and wireless energy transfer. Therefore, on the foundation of the S-ER relay protocol, we designed a novel two-phase ES-aided secure transmission scheme by jointly optimizing secure beamforming, ES, and TS coefficients to maximize the system confidentiality rate. Precisely, the main values of this essay are the following.

(1) A novel two-phase ES-aided secure transmission scheme is established by using the FD pattern to realize contemporaneous information as well as energy delivery. We study secure joint beamforming and ES and TS coefficient design for the SRM problem in FD-WPR systems;

(2) An antenna multiplexing method using the configured antenna to receive information to enhance the intensity of the information signal is proposed;

(3) To deal with the aforementioned difficult problem, one-dimensional (1D) search and semi-definite relaxation (SDR) techniques are used to resolve the question. Then, an alternative method to transform the original problem into two sub-problems is proposed. By solving these subproblems iteratively, the closed solution of the beamforming, the covariance of the ES, and the optimal time allocation are obtained. Moreover, the tightness of SDR is established with the aid of the Karysh-Kuhn-Tucker (KKT) conditions.

Section 2 describes the related work. Section 3 describes the system patterns and question formulation. Section 4 analyzes the secrecy rate optimization problem. Section 5 simulates and analyzes the performance of the suggested scheme. Section 6 concludes the paper.

\section{Related Work}

Simultaneous wireless information and power transfer (SWIPT) has been widely used in wireless communication networks [18,19], However, because of the openness of its energy collection, the security of its communication is threatened.

The SWIPT protocol's security is crucial given that SWIPT wireless networks are susceptible to eavesdropping due to the broadcasting and open characteristics of wireless media. The physical-layer (PHY) security technique that uses the inherent randomness of wireless channels is an efficient approach to improving the secrecy of wireless networks [20] establishes a FSK based swipt system powered by DC power supply for full duplex communication through shared channel. In [21], the end point node working in the fullduplex (FD) pattern in a single-input-single-output (SISO) SWIPT system, to obtain the highest security throughput of the system by a cooperative majorization of time switching (TS) coefficient, as well as codeword transmission rate, was studied. As an extension of [21], the authors of [22] investigated secrecy rate maximization (SRM), beamforming and null-space beamforming for secure communications in AF relay systems. The authors of [23] a scheme by which the destination is able to assist cooperative jamming to maximize the confidentiality rate. 
In [24,25], artificial noise (AN) is used to damage the channel quality of the eavesdropping channel. Meanwhile, collaborative relaying has been proven effective in spreading coverage and spatial diversity [26]. Moreover, studies have found that the relay also can enhance wireless PHY security $[5,6,8]$.

Based on the double advantages of AN and relay, a variety of secure transmission schemes were studied in $[27,28]$. Specifically, in [27], the authors proposed joint cooperative 45 beamforming $(\mathrm{CB})$ and an AN scheme for PHY confidentiality in AF multi-antenna multirelay systems. Additionally, as an extension of [27] introduced this scheme into SWIPT wireless networks. In [28], robust AN-aided secure beamforming was proposed to minimize the transmission power in wireless-energy relay systems. To ensure secure information delivery and power collecting, the robust and safe AN-assisted beamforming and powerallocation design were studied in the presence of imperfect channel state information (CSI) [27]. Despite having many advantages, the relay is "reluctant" to participate in information transmission due to its lack of energy.

For the above reasons, wireless-powered FD architecture has attracted increasingly more attention in studying secure systems. The FD model can increase the average secrecy rate by $33 \%-66 \%$, compared with the HD model. In [29], the authors suggested a fresh TSFD relaying plan to improve secure transmission in wireless-powered systems. Moreover, a cooperative jamming scheme with FD architecture under friendly jammers was proposed, in which jammer nodes transmit jamming signals to reduce the channel quality of an eavesdropper [30].

\section{System Pattern and Question Formulation}

We studied a typical FD-WPR system, as presented in Figure 1, in which the system consists of a source node (S), a relay node (R), an information receiver node (IR), numerous energy receiver nodes $\left(E R_{K}\right)$, and innumerable eavesdroppers (EMs). $S$ transmits confidential information to the IR, and $\left(\mathrm{ER}_{\mathrm{K}}\right)$ harvests energy with the help of a trusted relay under the existence of $\mathrm{E}_{\mathrm{M}}$. The relay has an energy harvester $(\mathrm{EH})$ and an information receiver $(\mathrm{Rx})$. Except for the relay, which has $N+1$ feeler, all nodes are installed with one antenna. Specifically, all the antennas in the relay are used to transmit information and harvest energy. Besides, we suppose that all channels are quasistatic block fading channels.

Moreover, the relay knows the channel state information of all channels. We adopted a two-phase TS secure relaying protocol to secure transmission and efficient wireless energy transfer in the system. For this two-phase protocol, the relay not only avoids selfinterference but also harvests energy from its message, as shown in Figure 2. In the first phase (see Figure 2a), at time slot n, one antenna is used to receive information transmitted from the $S$ at $R_{x}$, while $N$ antennas are used to harvest energy from the $S$ transmission at the EH. The received signals at the Rx and EH, as well as the harvested energy at the EH, are, respectively, given as:

$$
\begin{aligned}
y_{r, 1}^{R x}[n] & =h_{1} x_{S}[n]+n_{1}[n] \\
\mathbf{y}_{r, 1}^{E H}[n] & =\mathbf{h}_{2} x_{S}[n]+\mathbf{n}_{2}[n] \\
E_{1} & =\alpha T \eta P_{S}\left\|\mathbf{h}_{2}\right\|^{2},
\end{aligned}
$$

where $x_{S}[n]$ denotes the confidential information sent by $\mathrm{S}$, satisfying $\mathbb{E}\left|x_{S}[n]\right|^{2}=P_{S} ; h_{1}$ and $\mathbf{h}_{2}$ are the route factors from $S$ to the receiving antenna and $N$ transmitting antennas at $\mathrm{R} ; n_{1}$ and $\mathbf{n}_{2}$ denote the additive white Gaussian noise (AWGN) satisfying, and $n_{1}$ is used in Equation (1), and $\mathbf{n}_{2}$ is used in Equation (2). $\mathcal{C N}(0,1)$ at the Rx and $\mathcal{C N}\left(\mathbf{0}, \mathbf{I}_{N}\right)$ at the EH of $R ; \eta \in(0,1]$ is a continuous indicating power transfer effectiveness. 


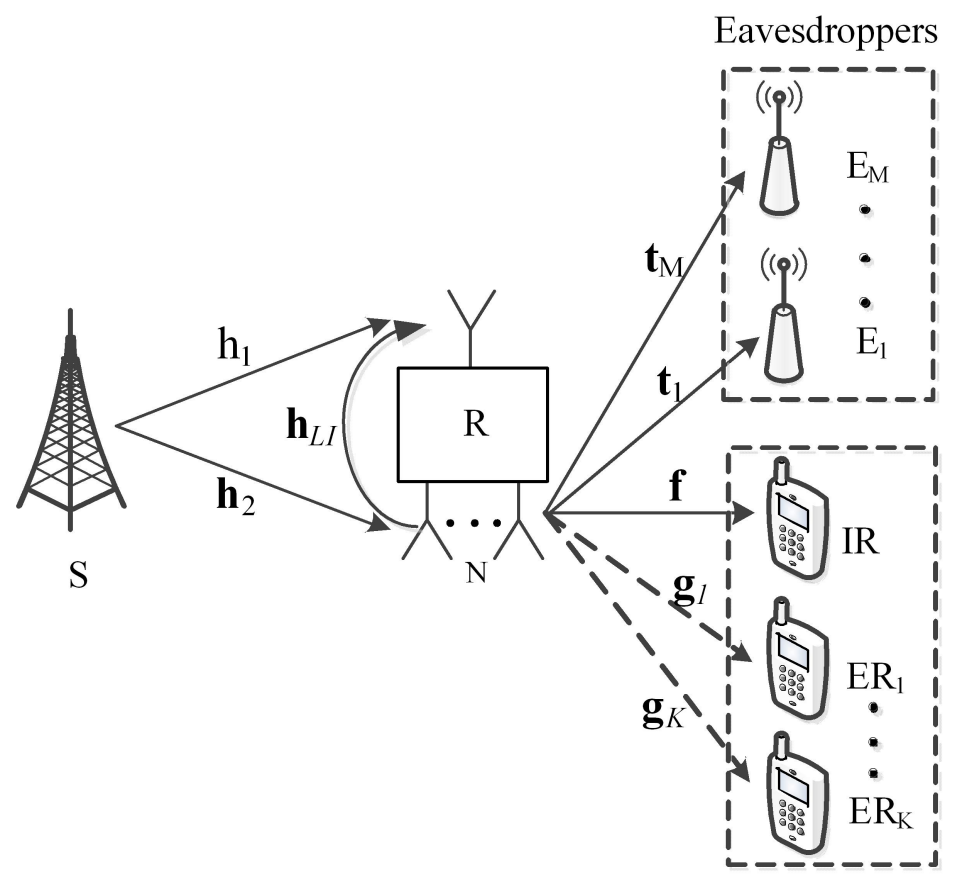

Legitimate users

Figure 1. System model of FD-WPR network.

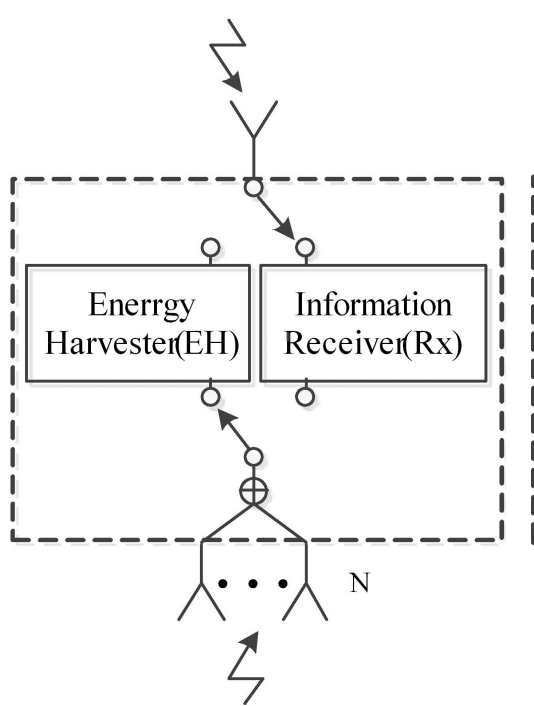

(a) Phase $1(\alpha T)$

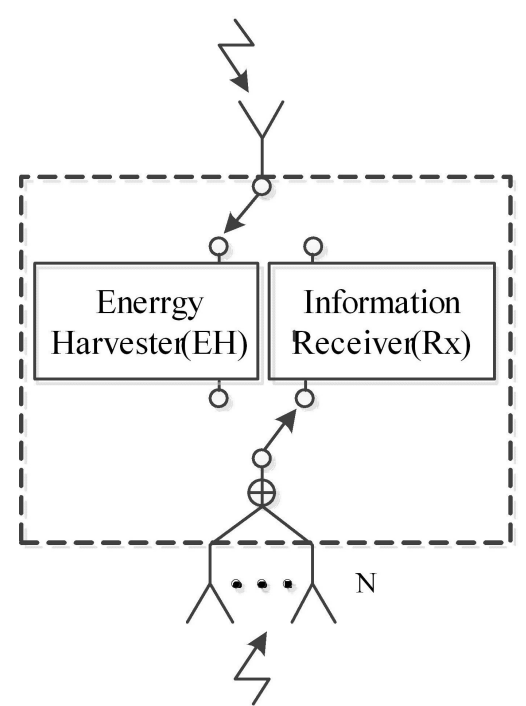

(b) Phase $2((1-\alpha) T)$

Figure 2. System model two-phase TS protocol for FD-WPR networks.

Under the second stage, as shown in Figure $2 \mathrm{~b}$, the $R x$ uses $N$ antennas to transmit the confidential information to legitimate users after relay processing, and the signal transmitted by the $R x$ to the IR with a superimposed ES is given by:

$$
\mathbf{x}_{r}[n]=y_{r, 1}^{R x}[n] \mathbf{w}+\mathbf{z}[n],
$$

where $\mathbf{w} \in \mathbb{C}^{N \times 1}$ represents the beamforming matrix at the $\mathrm{Rx} ; \mathbf{z}[n]$ denotes the ES phasor following $\mathbf{z}[n] \sim \mathcal{C N}(\mathbf{0}, \mathbf{\Sigma})$, and $\boldsymbol{\Sigma}$ is the covariance of the ES [5]. The processing delay at the 
relay is trivial compared to the transmission delay and thereby can be safely ignored [22]. At this moment, the transmit energy at $\mathrm{R}$ is provided by:

$$
P_{r}=P_{S}\left\|\mathbf{w} h_{1}\right\|^{2}+\|\mathbf{w}\|^{2}+\operatorname{Tr}(\boldsymbol{\Sigma}) .
$$

Meanwhile, the EH harvests two parts of the energy: one part is source information from S, and the other is self-power from the LI route. Consequently, the received signals and collected power at the $\mathrm{EH}$ is able to be written separately as:

$$
\begin{aligned}
& \mathbf{y}_{r, 2}^{E H}[n]=h_{1} x_{s}[n]+\mathbf{h}_{L I}^{H} \mathbf{x}_{r}[n]+n_{r}[n] \\
& E_{2}=(1-\alpha) T \eta\left(P_{s}\left|h_{1}\right|^{2}+P_{r}\left\|\mathbf{h}_{L I}^{H}\right\|^{2}\right),
\end{aligned}
$$

where $\mathbf{h}_{L I} \in \mathbb{C}^{N \times 1}$ is the $\mathrm{LI}$ route between feelers at the FD relay, which is beneficial and generally considered to be a Rayleigh fading channel [31]. Accordingly, this two-phase $\mathrm{S}$-ER protocol at $\mathrm{R}$ no longer requires additional power and hardware sources to hold LI back. In a unit time slot $\mathrm{T}$, the total collected power is computed as:

$$
\begin{aligned}
E & =E_{1}+E_{2} \\
& =\alpha T \eta P_{S}\left\|\mathbf{h}_{2}\right\|^{2}+(1-\alpha) T \eta\left(P_{S}\left|h_{1}\right|^{2}+P_{r}\left\|\mathbf{h}_{L I}^{H}\right\|^{2}\right) .
\end{aligned}
$$

By replacing (5) into (8), the energy harvested at $\mathrm{R}$ is able to be rewritten as:

$$
\begin{aligned}
E & =\alpha \operatorname{T\eta } P_{s}\left\|\mathbf{h}_{2}\right\|^{2}+(1-\alpha) \operatorname{T\eta }\left(P_{S}\left|h_{1}\right|^{2}\right. \\
& \left.+\left(P_{S}\left\|\mathbf{w} h_{1}\right\|^{2}+\|\mathbf{w}\|^{2}+\operatorname{Tr}(\boldsymbol{\Sigma})\right)\left\|\mathbf{h}_{L I}^{H}\right\|^{2}\right) .
\end{aligned}
$$

Therefore, the entire obtainable transmit energy of the relay is $(E / T)$, which is denoted:

$$
\begin{aligned}
P^{\max } & =\alpha \eta P_{S}\left\|\mathbf{h}_{2}\right\|^{2}+(1-\alpha) \eta\left(P_{S}\left|h_{1}\right|^{2}\right. \\
& \left.+\left(P_{s}\left\|\mathbf{w} h_{1}\right\|^{2}+\|\mathbf{w}\|^{2}+\operatorname{Tr}(\boldsymbol{\Sigma})\right)\left\|\mathbf{h}_{L I}^{H}\right\|^{2}\right) .
\end{aligned}
$$

As described above, the received signals at the IR, $k$ th $E R$, and $m$ th listener is able to be respectively formulated as:

$$
\begin{gathered}
y_{d}[n]=\mathbf{f}^{H} \mathbf{x}_{r}[n]+n_{3} \\
y_{e, k}[n]=\mathbf{g}_{k}^{H} \mathbf{x}_{r}[n]+n_{4}, \quad k=1, \ldots, K \\
y_{e, m}[n]=\mathbf{t}_{m}^{H} \mathbf{x}_{r}[n]+n_{5}, \quad m=1, \ldots, M,
\end{gathered}
$$

where $\mathbf{f} \in \mathbb{C}^{N \times 1}, \mathbf{g}_{k} \in \mathbb{C}^{N \times 1}$, and $\mathbf{t}_{m} \in \mathbb{C}^{N \times 1}$ represent route replies from the Rx to the IR, $\mathrm{ER}$, and $m$ th listener, separately; $n_{3}, n_{4}$, and $n_{5}$ denote the AWGN listed below $\mathcal{C N}(0,1)$, respectively.

From the system pattern (11)-(13), the obtained signal-to-noise ratio (SNR) at the IR, $\mathrm{m}$ th eavesdropper and ER harvested energy are derived as:

$$
\begin{gathered}
\gamma_{d}=\frac{\mathbf{w}^{H} \mathbf{A} \mathbf{w}}{\mathbf{w}^{H} \mathbf{B w}+1} \\
\gamma_{e, m}=\frac{\mathbf{w}^{H} \mathbf{A}_{e, m} \mathbf{w}}{\mathbf{w}^{H} \mathbf{B}_{e, m} \mathbf{w}+\mathbf{t}_{m}^{H} \boldsymbol{\Sigma} \mathbf{t}_{m}+1} \\
E_{k}=\eta(1-\alpha) T\left(\mathbf{w}^{H}\left(\mathbf{A}_{g}+\mathbf{B}_{g}\right) \mathbf{w}+\mathbf{g}_{k}^{H} \boldsymbol{\Sigma} \mathbf{g}_{k}\right),
\end{gathered}
$$

where $\mathbf{A}=P_{S}\left|h_{1}\right|^{2} \mathbf{D}(\mathbf{f}) \mathbf{D}^{H}(\mathbf{f}), \mathbf{B}=\mathbf{D}(\mathbf{f}) \mathbf{D}^{H}(\mathbf{f}), \mathbf{A}_{e, m}=P_{S}\left|h_{1}\right|^{2} \mathbf{D}\left(\mathbf{t}_{m}\right) \mathbf{D}^{H}\left(\mathbf{t}_{m}\right), \mathbf{B}_{e, m}=$ $\mathbf{D}\left(\mathbf{t}_{m}\right) \mathbf{D}^{H}\left(\mathbf{t}_{m}\right), \quad \mathbf{A}_{g}=P_{S}\left|h_{1}\right|^{2} \mathbf{D}\left(\mathbf{g}_{k}\right) \mathbf{D}^{H}\left(\mathbf{g}_{k}\right)$, and $\mathbf{B}_{g}=\mathbf{D}\left(\mathbf{g}_{k}\right) \mathbf{D}^{H}\left(\mathbf{g}_{k}\right)$. Therefore, the achievable confidentiality rate is written as: 


$$
\begin{gathered}
R_{s}=\min _{m \in M}\left\{\log \left(1+\gamma_{d}\right)-\log \left(1+\gamma_{e, m}\right)\right\} \\
\text { s.t. } \quad E_{k} / T \geq P_{\min , k}, P_{r} \leq P_{\max }, 0<\alpha<1,
\end{gathered}
$$

where $P_{\min , k} \geq 0$ is the threshold value of individual minimum power transmission required by ER. Our target is to maximize the confidentiality rate and transfer energy to the ER by cooperatively designing the beamforming phasor $\mathbf{w}$ at the same time, ES covariance $\Sigma$, and TS ratio $\alpha$, under the limitation of the transmit energy restriction at $\mathrm{R}$ and ER. Mathematically, the SRM question can be defined as:

$$
\begin{gathered}
\max _{\mathbf{w}, \boldsymbol{\Sigma} \geq \mathbf{0}, \alpha} \min _{m \in M}\left\{\log \left(1+\gamma_{d}\right)-\log \left(1+\gamma_{e, m}\right)\right\} \\
\text { s.t. } \eta(1-\alpha)\left(\mathbf{w}^{H}\left(\mathbf{A}_{g}+\mathbf{B}_{g}\right) \mathbf{w}+\mathbf{g}_{k}^{H} \boldsymbol{\Sigma} \mathbf{g}_{k}\right) \geq P_{\min , k}, k \in K \\
C+\mathbf{w}^{H} \mathbf{D w}+\mathbf{w}^{H} \mathbf{E w}+F \mathbf{h}_{L I}^{H} \boldsymbol{\Sigma} \mathbf{h}_{L I} \geq\|G \mathbf{w}\|^{2}+\operatorname{Tr}(\boldsymbol{\Sigma}) \\
0<\alpha<1,
\end{gathered}
$$

where $C=\alpha \eta P_{S} \mathbf{D}\left(\mathbf{h}_{2}\right) \mathbf{D}^{H}\left(\mathbf{h}_{2}\right)+(1-\alpha) \eta P_{S}\left|h_{1}\right|^{2} ; \mathbf{D}=(1-\alpha) \eta P_{S}\left|h_{1}\right|^{2} \mathbf{D}\left(\mathbf{h}_{L I}\right) \mathbf{D}^{H}\left(\mathbf{h}_{L I}\right)$; $\mathbf{E}=(1-\alpha) \eta \mathbf{D}\left(\mathbf{h}_{L I}\right) \mathbf{D}^{H}\left(\mathbf{h}_{L I}\right) ; F=(1-\alpha) \eta ; G=P_{S}\left|h_{1}\right|^{2}+1$.

\section{Secrecy Rate Optimization}

In this section, the original problem (18) is converted into two sub-problems with respect to $(\mathbf{w}, \boldsymbol{\Sigma})$ and $\alpha$. As for the first sub-problems, we will reset the problem (18) into a two-rank majorization problem to resolve $(\mathbf{w}, \boldsymbol{\Sigma})$. As for the second sub-problem, we will use a 1D search to solve $\alpha$.

\subsection{Beamforming Vector and Energy Signal Design}

For fixed $\alpha$, we reformulate the problem (18) into a two-level optimization problem. By bringing in a relaxation variable $\tau$, we modify question (18) as:

$$
\begin{gathered}
R_{S}^{*}=\max _{\mathbf{w}, \boldsymbol{\Sigma} \geq \mathbf{0}}\left\{\log \left(1+\gamma_{d}\right)-\log \left(1+\frac{1}{\tau}\right)\right\} \\
\text { s.t. } \quad \log \left(1+\gamma_{e, m}\right) \leq \log \left(\frac{1}{\tau}\right), m \in M \\
\eta(1-\alpha)\left(\mathbf{w}^{H}\left(\mathbf{A}_{g}+\mathbf{B}_{g}\right) \mathbf{w}+\mathbf{g}_{k}^{H} \boldsymbol{\Sigma} \mathbf{g}_{k}\right) \geq P_{\min }, k \in K \\
C+\mathbf{w}^{H} \mathbf{D} \mathbf{w}+\mathbf{w}^{H} \mathbf{E w}+F \mathbf{h}_{L I}^{H} \boldsymbol{\Sigma} \mathbf{h}_{L I} \geq\|G \mathbf{w}\|^{2}+\operatorname{Tr}(\boldsymbol{\Sigma}) .
\end{gathered}
$$

By following the same ideas in [5], the problem above can be transformed into a two-rank question in which the extrinsic layer is a single-variable majorization question with respect to (w.r.t.), for example:

$$
\begin{aligned}
R_{S}^{*}=\max _{\tau} \quad\left\{\log (1+v(\tau))-\log \left(\frac{1}{\tau}\right)\right\} \\
\text { s.t. } \quad \tau_{\min } \leq \tau \leq 1,
\end{aligned}
$$

and the intrinsic layer is a quadratic fractional question w.r.t. $\mathrm{w}$ and $\Sigma$ for fixed $\tau$, which is given by:

$$
\begin{aligned}
& v(\tau)=\max _{\mathbf{w}, \boldsymbol{\Sigma} \succeq \mathbf{0}} \frac{\mathbf{w}^{H} \mathbf{A w}}{\mathbf{w}^{H} \mathbf{B} \mathbf{w}+1} \\
& \text { s.t. } \quad 1+\frac{\mathbf{w}^{H} \mathbf{A}_{e, m} \mathbf{w}}{\mathbf{w}^{H} \mathbf{B}_{e, m} \mathbf{w}+\mathbf{t}_{m}^{H} \boldsymbol{\Sigma} \mathbf{t}_{m}+1} \leq \frac{1}{\tau}, m \in M \\
& \eta(1-\alpha)\left(\mathbf{w}^{H}\left(\mathbf{A}_{g}+\mathbf{B}_{g}\right) \mathbf{w}+\mathbf{g}_{k}^{H} \boldsymbol{\Sigma} \mathbf{g}_{k}\right) \geq P_{\min }, k \in K \\
& C+\mathbf{w}^{H} \mathbf{D w}+\mathbf{w}^{H} \mathbf{E w}+F \mathbf{h}_{L I}^{H} \boldsymbol{\Sigma} \mathbf{h}_{L I} \geq\|G \mathbf{w}\|^{2}+\operatorname{Tr}(\boldsymbol{\Sigma}) .
\end{aligned}
$$


Next, we determine the variation range of $1 \mathrm{D}$ variables in the outer-level problem. According to the constraints in (19b), it is straightforward to have $\tau \leq 1 \triangleq \tau_{\min }$. On the basis of the limitations in (19a), if we want to ensure a positive secrecy rate, the following relation must be satisfied:

$$
\tau \geq\left(1+\frac{\mathbf{w}^{H} \mathbf{A w}}{\mathbf{w}^{H} \mathbf{B w}+1}\right) \geq \lambda_{\min }\left(\mathbf{B}^{-1} \mathbf{A}\right) \triangleq \tau_{\min } .
$$

The essential value of the reformulation is that the extrinsic-layer question (22) is a single-variable majorization question which is resolved by using a 1D line search over $\tau$. Nevertheless, the intrinsic-rank question (21) is still non-salient. Next, we commit to resolving question (21) by utilizing an SDR method [32].

According to a standard routing of SDR [17], by defining $\mathbf{W}=\mathbf{w} \mathbf{w}^{H}, \mathbf{M}=\mathbf{h}_{L I} \mathbf{h}_{L I}^{H} \mathbf{J}_{m}=$ $\mathbf{t}_{m} \mathbf{t}_{m}^{H}$, and $\mathbf{G}_{k}=\mathbf{g}_{k} \mathbf{g}_{k}^{H}$, and dropping the rank-1 restriction level rank $(\mathbf{W}=1)$ for the moment, we gain the relaxation of the question (21):

$$
\begin{aligned}
& v_{\text {relax }}(\tau)=\max _{\mathbf{w} \succeq \mathbf{0}, \boldsymbol{\Sigma} \succeq \mathbf{0}} \frac{\operatorname{Tr}(\mathbf{A W})}{\operatorname{Tr}(\mathbf{B W})+1} \\
& \text { s.t. } \quad \operatorname{Tr}\left(\left(\mathbf{A}_{e, m}-\rho \mathbf{B}_{e, m}\right) \mathbf{W}\right)-\rho \operatorname{Tr}\left(\mathbf{J}_{m} \boldsymbol{\Sigma}\right) \leq \rho, m \in M \\
& \eta(1-\alpha)\left(\operatorname{Tr}\left(\left(\mathbf{A}_{g}+\mathbf{B}_{g}\right) \mathbf{W}\right)+\operatorname{Tr}\left(\mathbf{G}_{k} \boldsymbol{\Sigma}\right)\right) \geq P_{\text {min }}, k \in K \\
& \text { C }+\operatorname{Tr}(\mathbf{D W})+\operatorname{Tr}(\mathbf{E W})+F \operatorname{Tr}(\mathbf{M} \boldsymbol{\Sigma}) \geq \operatorname{Tr}(G \mathbf{W})+\operatorname{Tr}(\boldsymbol{\Sigma}),
\end{aligned}
$$

where $\rho=1 / \tau-1$. Then, we transform the quasi-salient question (23) into salient semidefinite programs (SDPs) using an efficient Charnes-Cooper transformation [27], notably making the variable substitutions $\overline{\mathbf{W}}=\beta \mathbf{W}, \overline{\boldsymbol{\Sigma}}=\beta \boldsymbol{\Sigma}$ by introducing a relaxation variable $\beta \geq 0$. We recast (23) as:

$$
\begin{aligned}
& \max _{\overline{\mathbf{W}} \succeq \mathbf{0}, \overline{\mathbf{\Sigma}} \succeq \mathbf{0}} \operatorname{Tr}(\mathbf{A} \overline{\mathbf{W}}) \\
& \text { s.t. } \quad \operatorname{Tr}(\mathbf{B} \overline{\mathbf{W}})+\beta=1 \\
& \operatorname{Tr}\left(\left(\mathbf{A}_{e, m}-\rho \mathbf{B}_{e, m}\right) \overline{\mathbf{W}}\right)-\rho \operatorname{Tr}\left(\mathbf{J}_{m} \overline{\boldsymbol{\Sigma}}\right) \leq \rho \beta, m \in M \\
& \eta(1-\alpha)\left(\operatorname{Tr}\left(\left(\mathbf{A}_{g}+\mathbf{B}_{g}\right) \overline{\mathbf{W}}\right)+\operatorname{Tr}\left(\mathbf{G}_{k} \overline{\boldsymbol{\Sigma}}\right)\right) \geq \beta P_{\min }, k \in K \\
& C \beta+\operatorname{Tr}(\mathbf{D} \overline{\mathbf{W}})+\operatorname{Tr}(\mathbf{E} \overline{\mathbf{W}})+F \operatorname{Tr}(\mathbf{M} \overline{\boldsymbol{\Sigma}}) \geq \operatorname{Tr}(G \overline{\mathbf{W}})+\operatorname{Tr}(\overline{\boldsymbol{\Sigma}}) .
\end{aligned}
$$

Question (24) is an SDP salient question, which is validly determined using standard optimization packages such as CVX [33]. At this point, problem (24) has been solved. We can obtain the optimum resolution of $\mathbf{W}^{*}$ and $\Sigma^{*}$ by the relations $\overline{\mathbf{W}}=\beta \mathbf{W}$ and $\bar{\Sigma}=\beta \Sigma$, respectively.

Because we dropped the non-convex rank-1 constraint, the search region of possible solutions is expanded. However, the question of whether the SDR is tight for question (21) remains. To resolve this problem, we suppose the energy minimization question listed in question (25).

$$
\begin{gathered}
\max _{\mathbf{W} \succeq \mathbf{0}, \overline{\boldsymbol{\Sigma}} \succeq \mathbf{0}} \operatorname{Tr}(\mathbf{G W}) \\
\text { s.t. } \quad \operatorname{Tr}\left(\left(\mathbf{A}_{e, m}-\rho \mathbf{B}_{e, m}\right) \mathbf{W}\right)-\rho \operatorname{Tr}\left(\mathbf{J}_{m} \boldsymbol{\Sigma}\right) \leq \rho, m \in M \\
\eta(1-\alpha)\left(\operatorname{Tr}\left(\left(\mathbf{A}_{g}+\mathbf{B}_{g}\right) \mathbf{W}\right)+\operatorname{Tr}\left(\mathbf{G}_{k} \boldsymbol{\Sigma}\right)\right) \geq P_{\min }, \quad k \in K \\
C+\operatorname{Tr}(\mathbf{D W})+\operatorname{Tr}(\mathbf{E} \mathbf{W})+F \operatorname{Tr}(\mathbf{M} \boldsymbol{\Sigma}) \geq \operatorname{Tr}(G \mathbf{W})+\operatorname{Tr}(\boldsymbol{\Sigma}) \\
\operatorname{Tr}\left(\left(\mathbf{A}-v_{\text {relax }}(\tau) \mathbf{B}\right) \mathbf{W}\right) \geq v_{\text {relax }}(\tau) .
\end{gathered}
$$

$v_{\text {relax }}(\tau)$ is the optimum value of question (24), which is a continuous in question (25). Constraint (25e) is derived from: $\frac{\operatorname{Tr}(\mathbf{A W})}{\operatorname{Tr}(\mathbf{B W})+1} \geq v_{\text {relax }}(\tau)$. Therefore, we have the following propositions: 
Thesis 1. Any practicable resolution to question (25) is the optimum resolution to question (23). The Evidence is similar to that in [7] [Thesis 2], and the Evidence thus left out here.

Thesis 2. For $v(\tau)>0$, any optimum $\mathbf{W}^{*} 132$ to the question (25) always meets level $\operatorname{rank}\left(\overline{\mathbf{W}^{*}}\right)=1$.

Proof. See Appendix A.1.

Thesis 3. Assume that the initial problem (21) is practicable $v(\tau)>0$. Subsequently, the optimal resolution can be gained by resolving the SDP problem (25). Moreover, the optimum resolution $\overline{\mathbf{W}^{*}}$ must be of level 1 .

Proof. Thesis 3 is a straightforward outcome of Propositions 1 and 2.

\subsection{Description of the Solving Algorithm}

We now return to the two-level optimization problems (20) and (21); the algorithm for resolving question (19) is specified in Algorithm 1.

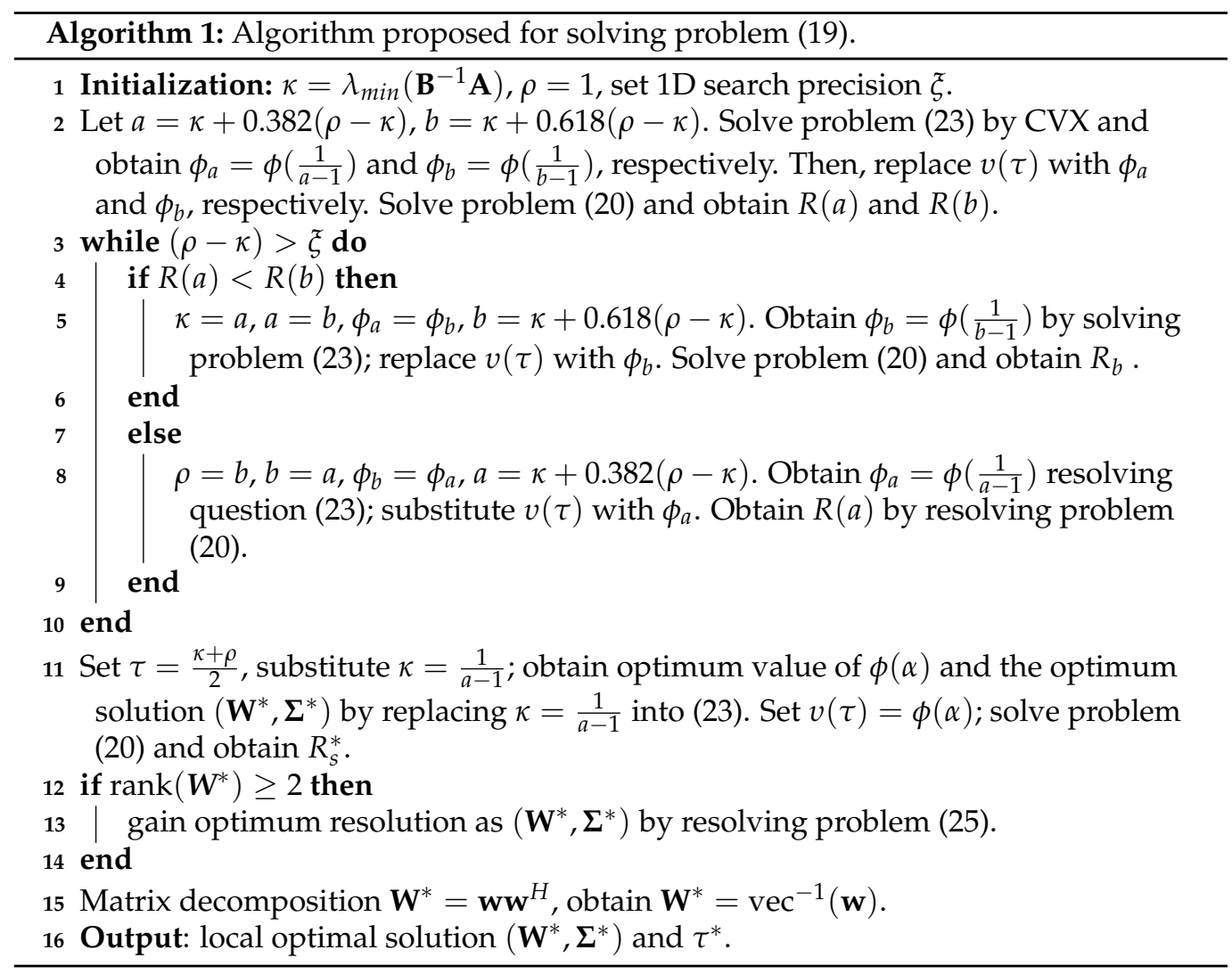

\subsection{Time Allocation Ratio Design}

We obtained the optimal solutions of $\left(\mathbf{W}^{*}, \boldsymbol{\Sigma}^{*}\right)$ and $\tau^{*}$. According to the idea of monotonicity between SNR and SRM, for given $\mathbf{W}^{*}, \Sigma^{*}$, and $\tau^{*}$, we are able to reorganize question (18) as:

$$
R_{S}^{*}=\max _{\alpha}\left\{\log \left(\frac{\mathbf{w}^{H} \mathbf{A} \mathbf{w}}{\mathbf{w}^{H} \mathbf{B w}+1}\right)-\log \left(\frac{\mathbf{w}^{H} \mathbf{A}_{e, m} \mathbf{w}}{\mathbf{w}^{H} \mathbf{B}_{e, m} \mathbf{w}+\mathbf{t}_{m}^{H} \mathbf{\Sigma} \mathbf{t}_{m}+1}\right)\right\} .
$$

In this way, we can obtain the optimum solution by utilizing a $1 \mathrm{D}$ examination so as to obtain the optimal target value $R^{*}$. 


\section{Numerical Results}

In this section, we inspect the property of the suggested scheme in FD-WPR systems by numerical imitations. Specifically, with the intention of making the advantage of the suggested scheme more obvious, we contrast the scheme with the benchmarks below: (1) No ES scheme; by setting the covariance matrix $\Sigma=\mathbf{0}$, we only optimize ww and $\alpha$ in (18); (2) AN scheme; the relay simultaneously forwards information and broadcasts AN, where AN cannot be eliminated at the destination. The Monte Carlo Imitation method is employed in order to verify the confidentiality rate of the proposed method. All of the numerical outcomes are the average of 1000 haphazardly produced channels. Unless otherwise noted, the simulation settings are the following: $P_{S}=10 \mathrm{dBW}, K=3, M=3$, $\eta=0.8, P_{\max }=20 \mathrm{dBW}, P_{\min }=3 \mathrm{dBW}$, and $N=3$.

The influence of transmission power $P_{S}$ on the confidentiality rate is shown in Figure 3. Naturally, the system confidentiality rate of all plans grows together with increasing $P_{S}$. It is seen that when $P_{S}$ is very small, the secrecy rate will increase rapidly. As $P_{S}$ increases, the secrecy rate continues to increase, but at a slow pace. Moreover, we also discover that the suggested ES plan outclasses the AN scheme, while the scheme without ES (no ES) performs the worst. Furthermore, it can be observed that the performance of ES-eve is close to ES-noeve. This phenomenon suggests that the legitimate channel will not be impaired by ES. Based on the above observations, we are confident in saying that ES is more appropriate for secure transmission than AN. The relay broadcasts AN to jam eavesdroppers but impairs the quality of the legitimate channel simultaneously.

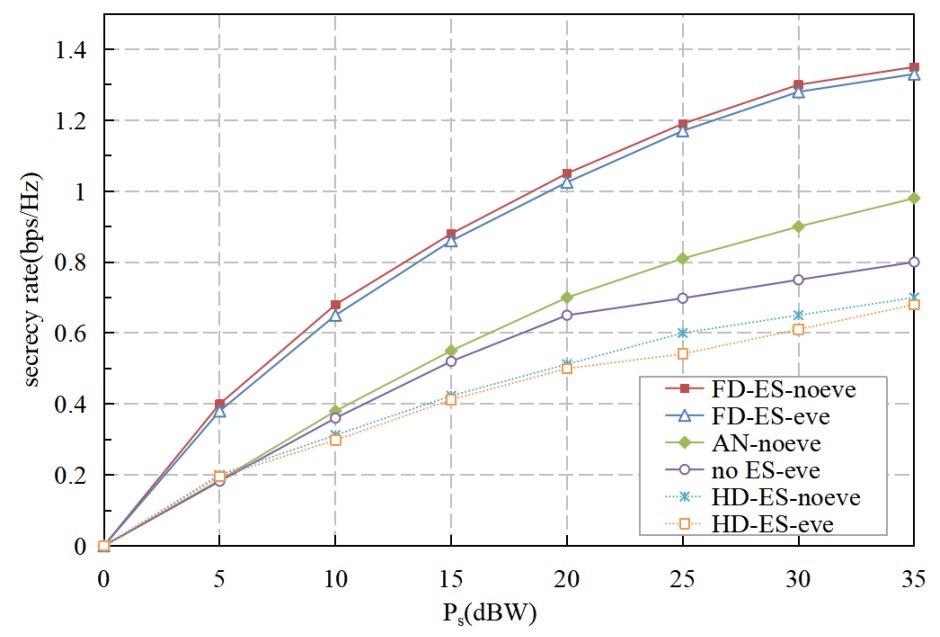

Figure 3. Secrecy rate vs. source transmission power.

Figure 4 presents the change of secrecy rate under diverse maximum available powers $P_{\max }$ at R. It is shown the system secrecy rate of all schemes increases with increasing $P_{\max }$, while the proposed ES scheme exhibits the best performance.The reason for the maximum safety rate of our scheme is that we have introduced self energy recovery technology, The energy signal we introduced effectively reduces the signal-to-noise ratio of eavesdropping nodes, so the security rate is higher than other methods. According to Formula (10), the greater $P_{\text {max }}$, the more energy collected at the relay, and the greater the transmission power of the relay. Therefore, according to Formulas (11) and (17), the greater the safety rate of the system. so the energy collected at the relay is higher than other schemes. That is, the transmission power of the relay is higher. When $P_{\max }<5 \mathrm{dBW}$, the performance of ES-noeve, ES-eve, and AN-eve are very similar. In addition, the performance of the ES-eve scheme is always able to approach that of the ES-noeve system. Furthermore, when $P_{\max }<15 \mathrm{dBW}$, the secrecy rate increases rapidly, whereas, when $P_{\max }>15 \mathrm{dBW}$, the secrecy rate increases slowly. This implies that the proposed ES schemes can support SWIPT transmission security without additional power. 


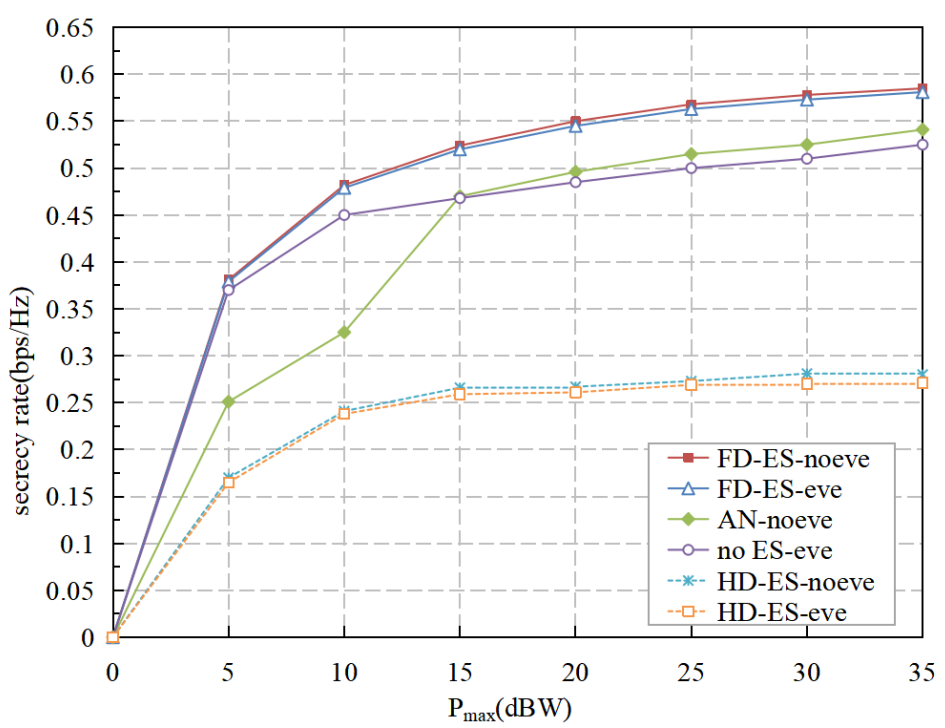

Figure 4. Secrecy rate vs. maximum available power at relay.

In Figure 5, we plot the confidentiality rate against the minimum required power threshold at ER. It is presented in Figure 4 that, when $P_{\min }<3 \mathrm{dBW}$, the system confidentiality rate is almost unchanged, whereas the system secrecy rate rapidly decreases when $P_{\min }>3 \mathrm{dBW}$. Moreover, by comparison with other plans, the suggested plan exhibits a meaningful performance boost with regard to the system secrecy rate. It is discovered that the ES-eve project's property can approach that of the ES-noeve program. No matter how $P_{\min }$ changes, the implementation of the ES-eve is close to that of ES-noeve. The performance of the no-ES-eve scheme is the worst, which proves the superiority of the suggested ES plan.

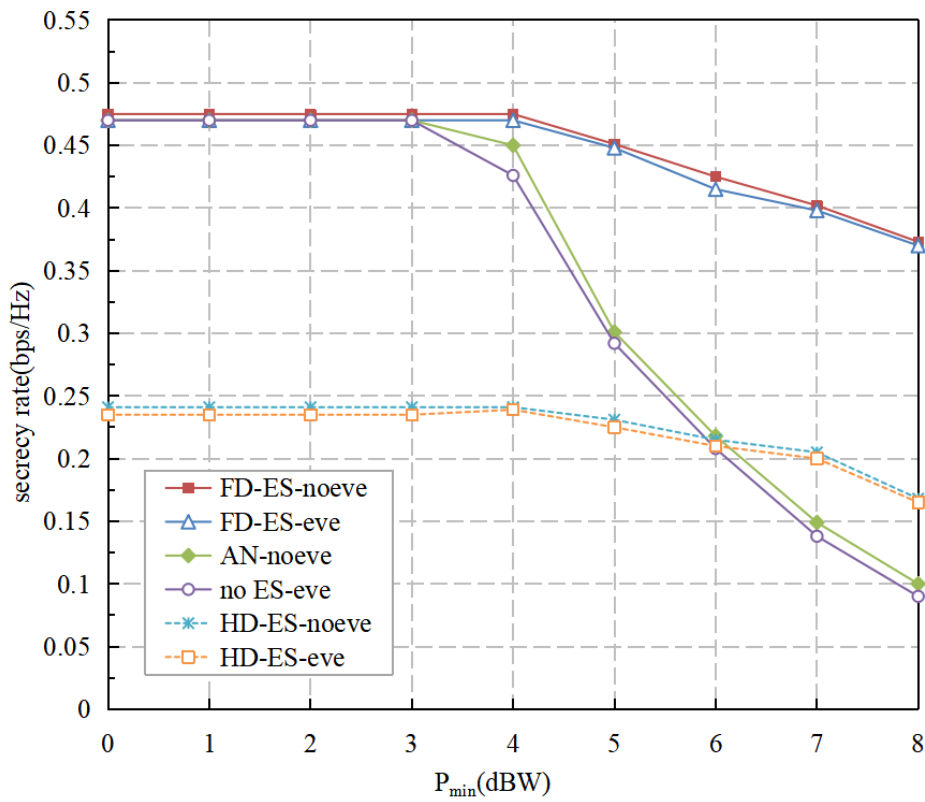

Figure 5. Secrecy rate vs. minimum essential energy at C.

To investigate the performance of the proposed ES scheme more comprehensively, Figure 6 shows the relationship between the energy conversion efficiency factor $\eta$ versus the 
secrecy rate. $\eta$ determines how much receiving power $\mathrm{R}$ can actually provide. According to Formulas (9), (16) and (17), when $P_{\text {min }}$ increases to a certain extent, the shorter the time for signal transmission, resulting in the reduction of the safety rate of the system. Thus, finally, the system confidentiality rate can be enhanced.However, more severe information disclosure will raise harvest energy, ultimately leading to the slow improvement of secrecy performance in the high- $\eta$ regime. In addition, compared with the AN scheme, the ES scheme significantly increases the secrecy rate. In addition, we apply the ES method to a half-duplex communication system [34,35]. The results show that the effect of a half-duplex is approximately half of that of a full-duplex. We believe that the energy captured by the half-duplex mechanism is halved in the working process.

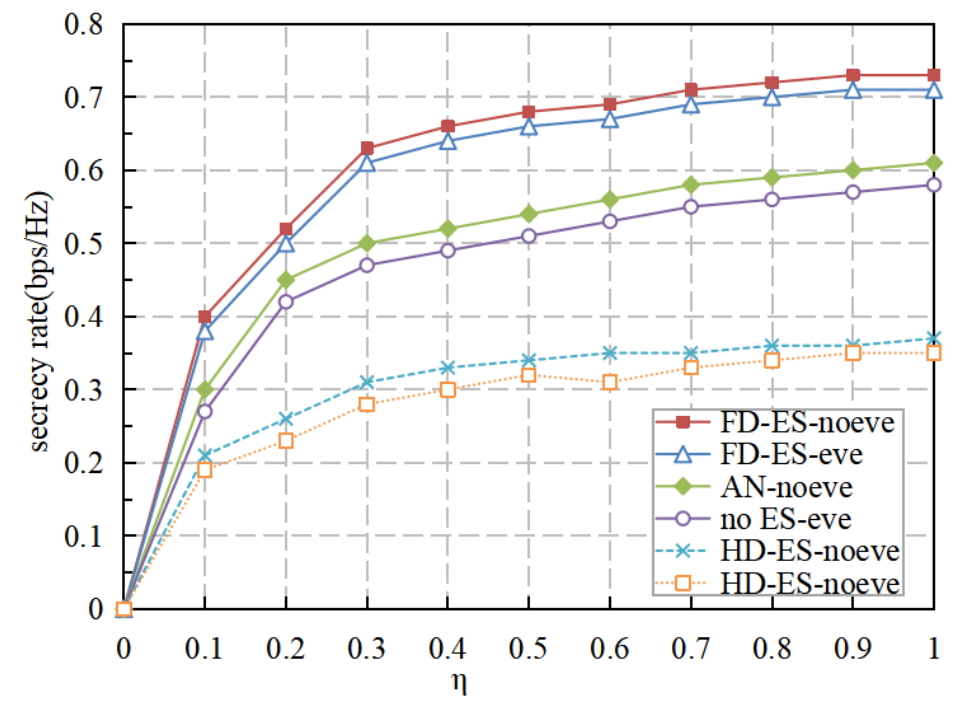

Figure 6. Secrecy rate vs. energy harvesting efficiency.

\section{Conclusions}

In this paper, on the foundation of the self-power circulation relay agreement, we propose a joint ES-aided secure beamforming and TS scheme in FD-WPR networks in which multiple eavesdroppers exist. In such conditions, this essay aims to resolve the SRM question of the relevant systems. We formulate the optimization problem by jointly optimizing ES covariances, AF beamforming, and TS coefficients. Since the formulated question is non-salient and challenging to deal with directly, we turn it into two subproblems. A key component of our method is to determine the convexity of some hidden SRM problems by using an SDR technique. Moreover, a relaxation problem always exists for which an optimal solution of rank 1 has been proven. Imitation outcomes demonstrate the advantage of the suggested ES scheme [36].

Author Contributions: L.O. Conceptualization, software and writing—original draft; P.C. methodology, investigation, formal analysis and writing - review and editing; X.L. Project administration and supervision; S.D. validation and resources; Y.S. data curation and visualization. All authors have read and agreed to the published version of the manuscript.

Funding: This research received no external funding.

Institutional Review Board Statement: Not applicable.

Informed Consent Statement: Not applicable.

Data Availability Statement: Not applicable.

Conflicts of Interest: The authors declare no conflict of interest. 


\section{Appendix A}

Appendix A.1. Proof of Proposition 2

Since (25) is salient and meets Slater's restriction condition, its dual gap is 0, and there is a strong dichotomy between the primitive and double problems. According to the optimal solution satisfying the KKT condition, we have

$$
\begin{aligned}
L(x)= & \operatorname{Tr}(G \mathbf{W})+\sum_{m=1}^{M} \lambda_{m}\left(\operatorname{Tr}\left(\left(\mathbf{A}_{e, m}-\rho \mathbf{B}_{e, m}\right) \mathbf{W}\right)-\rho \operatorname{Tr}\left(\mathbf{J}_{m} \boldsymbol{\Sigma}\right)\right. \\
& -\rho)+\sum_{k=1}^{K} \theta_{k}\left(\operatorname{Tr}\left(\left(\mathbf{A}_{g}+\mathbf{B}_{g}\right) \mathbf{W}\right)+\operatorname{Tr}\left(\mathbf{G}_{k} \boldsymbol{\Sigma}\right)-P_{\min }\right) \\
& +\mu(C+\operatorname{Tr}(\mathbf{D W})+\operatorname{Tr}(\mathbf{E W})+F \operatorname{Tr}(\mathbf{M} \boldsymbol{\Sigma})-\operatorname{Tr}(G \mathbf{W}) \\
& -\operatorname{Tr}(\boldsymbol{\Sigma}))-\omega\left(\operatorname{Tr}\left(\left(\mathbf{A}-v_{\text {relax }}(\tau) \mathbf{B}\right) \mathbf{W}\right)-v_{\text {relax }}(\tau)\right) \\
& -\operatorname{Tr}(\mathbf{Z W})-\operatorname{Tr}(\mathbf{L} \boldsymbol{\Sigma})
\end{aligned}
$$

where $\lambda_{m} \geq 0, \forall k, \mu \geq 0, \omega \geq 0, \mathbf{Z} \succeq \mathbf{0}$, and $\mathbf{L} \succeq \mathbf{0}$ are the Lagrangian multipliers associated with constraints (25b)-(25e), respectively. The proofs of the KKT conditions of the problem of (21) are expressed as follows:

$$
\begin{gathered}
\mathbf{Z W}=\mathbf{0} \text { and } \mathbf{L} \boldsymbol{\Sigma}=\mathbf{0} \\
\mathbf{Z}=(1-\mu) G+\sum_{m=1}^{M} \lambda_{m}\left(\mathbf{A}_{e, m}-\rho \mathbf{B}_{e, m}\right) \\
+\sum_{k=1}^{K} \theta_{k}\left(\mathbf{A}_{g}+\mathbf{B}_{g}\right)+\mu(\mathbf{D}+\mathbf{E})-\omega\left(\mathbf{A}-v_{\text {relax }}(\tau) \mathbf{B}\right) \\
\mathbf{L}=-\sum_{m=1}^{M} \lambda_{m} \rho \mathbf{J}_{m} \boldsymbol{\Sigma}+\sum_{k=1}^{K} \theta_{k} \mathbf{G}_{k}+\mu\left(F \mathbf{M}-\mathbf{I}_{K}\right)
\end{gathered}
$$

Note from (29) the oblique part of $\mathbf{L}$ can be formulated as

$$
\operatorname{diag}(\mathbf{L})=-\sum_{m=1}^{M} \lambda_{m} \rho \mathbf{J}_{m} \boldsymbol{\Sigma}+\sum_{k=1}^{K} \theta_{k} \mathbf{B}_{g}+\mu\left(\mathbf{E}-\mathbf{I}_{K}\right)
$$

Hence, (30) minus (28) can can be formulated as

$$
\begin{aligned}
\mathbf{Z} & =(1-\mu) G+\sum_{m=1}^{M} \lambda_{m}\left(\mathbf{A}_{e, m}-\rho \mathbf{B}_{e, m}\right) \\
& +\sum_{k=1}^{K} \theta_{k} \mathbf{A}_{g}+\mu \mathbf{D}-\omega \mathbf{A}+v_{\text {relax }}(\tau) \mathbf{B}
\end{aligned}
$$

In addition, according to the post-multiply property and (28), we can derive

$$
\omega \mathbf{A W}=\underbrace{\left.(1-\mu) G+\sum_{m=1}^{M} \lambda_{m}\left(\mathbf{A}_{e, m}-\rho \mathbf{B}_{e, m}\right)+\sum_{k=1}^{K} \theta_{k} \mathbf{A}_{g}+\mu \mathbf{D}+\vartheta_{\text {relax }}(\tau) \mathbf{B}\right) \mathbf{W}}_{\mathbf{Q}}
$$

Note that all of the other terms $\mathbf{Q}$ are semi-positive definite matrices, and thereby $\mathbf{Q} \succ \mathbf{0}$ is a full-rank matrix. Thus,

$$
\operatorname{rank}(\mathbf{W})=\operatorname{rank}(\mathbf{Q W})=\omega \operatorname{rank}(\mathbf{A W}) \leq \operatorname{rank}(\mathbf{A})=1
$$


where we find that $\operatorname{rank}(\mathbf{A})=1$ is based on $\operatorname{rank}(\mathbf{B} \otimes \mathbf{C})$. If $\operatorname{rank}(\mathbf{A}=\mathbf{0}$, then $\mathbf{A}=\mathbf{0}$, since there is a positive secrecy rate, namely $\vartheta_{\text {relax }}(\tau)>0$, and the constraint (25e) cannot be satisfied. Therefore, $\operatorname{rank}(\mathbf{A})=1$, which finalizes the evidence of Proposition 2.

\section{Appendix A.2. Notation Description}

\begin{tabular}{lll}
\hline No. & Notation & Description \\
\hline 1 & $\mathbf{A}^{H}$ & conjugate transpose of matrices A \\
2 & $\operatorname{Tr}(\mathrm{A})$ & trace of matrices A \\
3 & $\operatorname{rank}(\mathbf{A})$ & rank of matrices A \\
4 & $a=\operatorname{vec}(\mathbf{A})$ & stacking the columns of a matrices $\{\mathrm{A}\}$ into a phasor a \\
5 & $|\cdot|,\|\cdot\|$ and $\|\cdot\|_{F}$ & absolute value, Euclidean norm and Frobenius norm respectively \\
6 & $\otimes$ & Hadamard product \\
7 & $\mathbf{A} \succeq \mathbf{0}$ & $\{\mathrm{A}\}$ is a positive semi-definite matrices \\
8 & $\mathbf{I}_{N}$ & $N \times N$ identity matrix \\
9 & $\mathbf{D}(a)$ & the oblique matrices with $\partial$ vector on main oblique \\
10 & $\lambda_{\min (.)}$ & the minimum eigenvalue of the matrices involved \\
11 & $\chi \sim C N\left(\mu, \sigma^{2}\right)$ & a random variable abiding a complicated circular Gaussian distribution \\
& & with average $\mu$ as well as covariance $\sigma^{2}$ \\
\hline
\end{tabular}

\section{References}

1. Zhang, R.; Ho, C.K. MIMO Broadcasting for Simultaneous Wireless Information and Power Transfer. IEEE Trans. Wirel. Commun. 2013, 12, 1989-2001. [CrossRef]

2. Ng, D.W.K.; Lo E.S.; Schober, R. Robust Beamforming for Secure Communication in Systems With Wireless Information and Power Transfer. IEEE Trans. Wirel. Commun. 2014, 13, 4599-4615. [CrossRef]

3. Zhu, Z.; Zhang, Y.; Wang, Z.; Zhang, Y.; Gao, X.; Chu, Z. Robust beamforming for multiuser MISO interference channel with energy harvesting constraint. In Proceedings of the International Conference on Communications \& Networking in China, Shanghai, China, 15-17 August 2015.

4. Shi, Q.; Xu, W.; Wu, J.; Song, E.; Wang, Y. Secure Beamforming for MIMO Broadcasting With Wireless Information and Power Transfer. IEEE Trans. Wirel. Commun. 2015, 14, 2841-2853. [CrossRef]

5. Feng, Y.; Yang, Z.; Zhu, We.; Li, Q.; Lv, B. Robust Cooperative Secure Beamforming for Simultaneous Wireless Information and Power Transfer in Amplify-and-Forward Relay Networks. IEEE Trans. Veh. Technol. 2016, 66, 2354-2366. [CrossRef]

6. Wen, Z.; Liu, X.; Beaulieu, N.C.; Wang, R.; Wang, S. Joint Source and Relay Beamforming Design for Full-Duplex MIMO AF Relay SWIPT Systems. IEEE Commun. Lett. 2016, 20, 320-323. [CrossRef]

7. Wan, Y.; Li, Q.; Zhang, Q.; Qin, J. Optimal and Suboptimal Full-Duplex Secure Beamforming Designs for MISO Two-Way Communications. IEEE Wirel. Commun. Lett. 2015, 4, 493-496. [CrossRef]

8. Li, Q.; Zhang, Q.; Qin, J. Secure Relay Beamforming for SWIPT in Amplify-and-Forward Two-Way Relay Networks. IEEE Trans. Veh. Technol. 2016, 65, 9006-9019. [CrossRef]

9. Sabharwal, A.; Schniter, P.; Guo, D.; Bliss, D.W.; Rangarajan, S.; Wichman, R. In-Band Full-Duplex Wireless: Challenges and Opportunities. IEEE J. Sel. Areas Commun. 2014, 32, 1637-1652. [CrossRef]

10. Zheng, G. Joint Beamforming Optimization and Power Control for Full-Duplex MIMO Two-way Relay Channel. IEEE Trans. Signal Process. 2014, 63, 555-566. [CrossRef]

11. Zeng, Y.; Zhang, R. Full-Duplex Wireless-Powered Relay With Self-Energy Recycling. IEEE Wirel. Commun. Lett. 2015, 4, 201-204. [CrossRef]

12. Kim, H.; Kang, J.; Jeong, S.; Lee, K.E.; Kang, J. Secure beamforming and self-energy recycling with full-duplex wireless-powered relay. In Proceedings of the 2016 13th IEEE Annual Consumer Communications \& Networking Conference (CCNC), Las Vegas, NV, USA, 9-12 Januray 2016. [CrossRef]

13. Li, T.; Chen, P.; Wang, Z.; Xiao, N. Secure Beamforming for Simultaneous Wireless Information and Power Transfer in WirelessPowered Full-Duplex Relay Networks. In Proceedings of the 2019 14th International Conference on Computer Science \& Education (ICCSE), Toronto, ON, Canada, 19-21 August 2019.

14. Chen, P.; Li, T.; Fang, X. Secure Beamforming and Artificial Noise Design in Full-Duplex Wireless-Powered Relay Networks. In Proceedings of the 2019 IEEE 11th International Conference on Communication Software and Networks (ICCSN), Chongqing, China, 12-15 June 2019.

15. Wu, W.; Wang, B.; Deng, Z.; Zhang, H. Secure Beamforming for Full-Duplex Wireless Powered Communication Systems With Self-Energy Recycling. IEEE Wirel. Commun. Lett. 2017, 6, 146-149. [CrossRef]

16. $\mathrm{Hu}, \mathrm{S}$; Ding, Z.; Ni, Q. Beamforming optimisation in energy harvesting cooperative full-duplex networks with self-energy recycling protocol. IET Commun. 2016, 10, 848-853. [CrossRef] 
17. Su, Y.; Jiang, L.; He, C. Decode-and-forward relaying with full-duplex wireless information and power transfer. IET Commun. 2017, 11, 2110-2115. [CrossRef]

18. Chu, Z.; Zhou, F.; Xiao, P.; Zhu, Z.; Mi, D.; Al-Dhahir, N.; Tafazolli, R. Resource Allocation for Secure Wireless Powered Integrated Multicast and Unicast Services with Full Duplex Self-Energy Recycling. IEEE Trans. Wirel. Commun. 2018, 18, 620-636. [CrossRef]

19. Li, J.; Ding, X.; Li, X.; Zhao, K.; Shi, W. Research on the Energy Allocation Scheme Based on SWIPT Relaying System. Mob. Netw. Appl. 2018, 23, 1449-1458. [CrossRef]

20. Wu, J.; Kong, W.; Gao, P.; Jin, N.; Zhang, J.; Tao, J.; Snášel, V. Design Consideration of Bidirectional Wireless Power Transfer and Full-Duplex Communication System via a Shared Inductive Channel. Energies 2021, 14, 4918. [CrossRef]

21. Mou, W.; Cai, Y.; Yang, W.; Yang, W.; Xu, X.; Hu, J. Exploiting full Duplex techniques for secure communication in SWIPT system In Proceedings of the 2015 International Conference on Wireless Communications \& Signal Processing (WCSP), Nanjing, China, 15-17 October 2015.

22. Deng, Z.; Gao, Y.; Li, W.; Cai, C. Robust secure beamforming for SWIPT systems with full-duplex receivers and energy-harvesting eavesdroppers. In Proceedings of the 2017 IEEE International Conference on Consumer Electronics-Taiwan (ICCE-TW), Taipei, Taiwan, 12-14 June 2017.

23. Liu, M.; Liu, Y. Power Allocation for Secure SWIPT Systems with Wireless-Powered Cooperative Jamming. IEEE Commun. Lett. 2017, 21, 1353-1356. [CrossRef]

24. Niu, H.H.; Zhang, B.N.; Guo, D. Joint cooperative beamforming and artificial noise design for secure AF relay networks with energy-harvesting eavesdroppers. Front. Inf. Technol. Electron. Eng. 2017, 18, 850-862. [CrossRef]

25. Yu, H.; Wan, S.; Cai, W.; Xu, L.; Zhou, X.; Wang, J.; Wu, Y.; Shu, F.; Wang, J.; Wang, J. GPI-based Secrecy Rate Maximization Beamforming Scheme for Wireless Transmission with AN-aided Directional Modulation. IEEE Access 2017, 6, 12044-12051. [CrossRef]

26. Dang, S.; Chen, G.; Coon, J.P. Outage Performance Analysis of Full-Duplex Relay-Assisted Device-to-Device Systems in Uplink Cellular Networks. IEEE Trans. Veh. Technol. 2016, 99, 4506-4510. [CrossRef]

27. Hehao, N.; Zhang, B.; Guo, D.; Huang, Y. Joint Robust Design for Secure AF relay Networks with SWIPT. IEEE Access 2017. [CrossRef]

28. Li, B.; Fei, Z.; Chen, H. Robust Artificial Noise-Aided Secure Beamforming in Wireless-Powered Non-Regenerative Relay Networks. IEEE Access 2016, 4, 7921-7929. [CrossRef]

29. Qiao, J.; Zhang, H.; Zhou, X.; Yuan, D. Joint beamforming and time switching design for secrecy rate maximization in wirelesspowered FD relay systems. IEEE Trans. Veh. Technol. 2017, 67, 567-579. [CrossRef]

30. Bi, Y.; Chen, H. Accumulate and Jam: Towards Secure Communication via A Wireless-Powered Full-Duplex Jammer. IEEE J. Sel. Top. Signal Process. 2016, 10, 1538-1550. [CrossRef]

31. Qiao, J.; Zhang, H.; Zhao, F.; Yuan, D. Secure Transmission and Self-Energy Recycling with Partial Eavesdropper CSI. IEEE J. Sel. Areas Commun. 2018, 36, 1531-1543. [CrossRef]

32. Luo, Zh.; Ma, Wi.; So, A.Ma.; Ye, Y.; Zhang, S. Semidefinite Relaxation of Quadratic Optimization Problems. IEEE Signal Process. Mag. 2010, 27, 20-34. [CrossRef]

33. Michael Grant and Stephen Boyd. CVX: Matlab Software for Disciplined Convex Programming, Version 2.0 Beta. Available online: http:/ / cvxr.com/cvx (accessed on 20 September 2013).

34. Nguyen, T.N.; Tran, P.T.; Voznak, M. Wireless energy harvesting meets receiver diversity: A successful approach for two-way half-duplex relay networks over block Rayleigh fading channel. Comput. Netw. 2020, 172, 107176. [CrossRef]

35. Srirutchataboon, G.; Kochi, J.; Sugiura, S. Performance Analysis of Hybrid Buffer-Aided Cooperative Protocol Based on HalfDuplex and Virtual Full-Duplex Relay Selections. IEEE Open J. Commun. Soc. 2021, 2, 1862-1873. [CrossRef]

36. Zhu, G.; Lin, X.; Zhu, K.; Zhang, W.; Yu, J.X. TreeSpan: efficiently computing similarity all-matching. In Proceedings of the 2012 ACM SIGMOD International Conference on Management of Data, New York, NY, USA, 20-24 May 2012; pp. 529-540. [CrossRef] 